\title{
Families of Pisot numbers with negative trace
}

\author{
by \\ JAMES MCKEE (Oxford)
}

1. Introduction. A Pisot number is a real algebraic integer, $\theta$, such that $\theta>1$ and all conjugates of $\theta$ (other than $\theta$ itself) have modulus less than 1. The set of all Pisot numbers is usually denoted $S$ (after Salem).

Suppose that $r, k, a_{1}, \ldots, a_{r-k}$ are all integers with $r \geq 2,0 \leq k \leq r$, $a_{i} \geq 2(1 \leq i \leq r-k)$. If $r=2$ and $k=0$, then we exclude $a_{1}=a_{2}=2$. Then it was shown in [1] that the only roots of the equation

$$
\sum_{i=1}^{r-k} \frac{z^{a_{i}-1}-1}{z^{a_{i}}-1}+\frac{k}{z}=1
$$

are a certain Pisot number $\theta_{r, k}\left(a_{1}, \ldots, a_{r-k}\right)$, say, and its conjugates. Let $U$ be the set of all such Pisot numbers ( $T$ being used for Salem numbers!). Then (see [1])

- $U$ is a proper subset of $S$;

- trace $: U \rightarrow \mathbb{Z}$ is surjective.

In particular, there exist Pisot numbers of negative trace.

Indeed a construction was given in [1] which could produce Pisot numbers of any desired trace. Unfortunately, to produce negative trace the construction required that the degree of the Pisot number should be huge. An example was given (not claimed to be best-possible!) with trace -5 and degree 141731565070951.

In this paper it is shown how to construct Pisot numbers with negative trace and much smaller degree: the current record is 23837 . This cannot be too far from minimal for elements of $U$, in that a key result of this paper is that for minimality we may assume that each $a_{i}$ is a product of at least four distinct prime factors. It is hoped that a second, more computational, paper will establish several other extremal results. The ultimate goal is to find the smallest degree of any element of $S$ with negative trace, and finding

2000 Mathematics Subject Classification: Primary 11R06. 
the minimal degree for elements of $U$ with negative trace would be a step along the way.

2. Formulas for the degree and trace. To compute the minimal polynomial of $\theta_{r, k}\left(a_{1}, \ldots, a_{r-k}\right)$ we need to clear denominators in (1). If we multiply (1) by the $d$ th cyclotomic polynomial for every $d>1$ dividing one of the $a_{i}$, and multiply by $z$ if $k>0$, then the denominators will have been cleared. It was shown in [1], by computing residues at all relevant $d$ th roots of 1 , that nothing less will do. Hence we can read off formulas for the degree and trace:

$$
\operatorname{degree}\left(\theta_{r, k}\right)=\sum_{1<d \mid a_{i} \text { for some } i} \varphi(d)+\varepsilon,
$$

where

and

$$
\varepsilon= \begin{cases}0 & \text { if } k=0 \\ 1 & \text { if } k>0\end{cases}
$$

$$
\operatorname{trace}\left(\theta_{r, k}\right)=r+\sum_{1<d \mid a_{i} \text { for some } i} \mu(d) .
$$

Here $\varphi$ and $\mu$ are Euler's totient function and the Möbius function respectively.

From (2) and (3) we see immediately that in seeking negative trace with minimal degree we must have $k=0$. From now on we restrict to $k=0$, and write $\theta_{r}\left(a_{1}, \ldots, a_{r}\right)$ for $\theta_{r, 0}\left(a_{1}, \ldots, a_{r}\right)$. We shall now always have $\varepsilon=0$ in (2).

Applying inclusion-exclusion to (2) gives an alternative degree formula, which may be easier to use for computations:

$$
\begin{aligned}
\operatorname{degree}\left(\theta_{r}\right)= & -1+\sum_{i} a_{i}-\sum_{i<j} \operatorname{gcd}\left(a_{i}, a_{j}\right) \\
& +\sum_{i<j<k} \operatorname{gcd}\left(a_{i}, a_{j}, a_{k}\right)-\ldots
\end{aligned}
$$

Similarly we get a second formula for the trace:

$$
\operatorname{trace}\left(\theta_{r}\right)=\sum_{S \subseteq\left\{a_{1}, \ldots, a_{r}\right\},|S| \geq 2, \operatorname{gcd}(S)>1}(-1)^{|S|},
$$

where $\operatorname{gcd}(\{x, y, z, \ldots\})$ means $\operatorname{gcd}(x, y, z, \ldots)$.

Suppose that there are $n$ distinct primes dividing $a_{1}, \ldots, a_{r}$. Then (3) may be written

$$
\operatorname{trace}\left(\theta_{r}\right)=r-n+\sum_{d \mid a_{i} \text { for some } i, \omega(d) \geq 2} \mu(d),
$$

where $\omega(d)$ is the number of distinct prime factors of $d$. 
We glean two obvious minimality conditions from these formulas. Suppose that $\theta_{r}\left(a_{1}, \ldots, a_{r}\right)$ has minimal degree amongst elements of $U$ with negative trace. Then from (3) and (2) we see that

Each $a_{i}$ is squarefree.

Moreover (3) shows that the trace depends only on the pattern of the primes dividing $a_{1}, \ldots, a_{r}$, not on the primes themselves. Hence from (2) we find that for minimality

$$
\begin{aligned}
& \text { If } p_{1}, \ldots, p_{n} \text { are the distinct primes dividing } a_{1}, \ldots, a_{r}, \\
& \text { then } p_{1}, \ldots, p_{n} \text { are the first } n \text { primes in some order. }
\end{aligned}
$$

In what follows, we shall suppose that (7) and (8) always hold.

3. Dual Pisot numbers. Suppose that $\theta_{r}\left(a_{1}, \ldots, a_{r}\right) \in U$, with $\theta_{r}\left(a_{1}, \ldots, a_{r}\right)$ satisfying $(7)$ and $(8)$. Thus $p_{1}, \ldots, p_{n}$ are the first $n$ primes in some order. Let $q_{1}, \ldots, q_{r}$ be the first $r$ primes. We define the dual of $\theta_{r}\left(a_{1}, \ldots, a_{r}\right)$ to be $\theta_{n}\left(b_{1}, \ldots, b_{n}\right)$, where $b_{j}(1 \leq j \leq n)$ is the product of those $q_{i}$ for which $a_{i}$ is divisible by $p_{j}$. Note that the dual Pisot number does not depend on the ordering of $p_{1}, \ldots, p_{n}$ : changing the order merely has the affect of permuting $b_{1}, \ldots, b_{n}$, which does not change $\theta_{n}\left(b_{1}, \ldots, b_{n}\right)$.

For example, if $r=3, n=4$, then the dual of $\theta_{3}\left(p_{1} p_{2} p_{3}, p_{1} p_{2}, p_{3} p_{4}\right)$ is $\theta_{4}\left(q_{1} q_{2}, q_{1} q_{2}, q_{1} q_{3}, q_{3}\right)$. (We have $b_{1}=q_{1} q_{2}$ since $p_{1}$ divides $a_{1}$ and $a_{2}$, etc. If we chose to swap the labels $p_{3}$ and $p_{4}$, then the dual would be $\theta_{4}\left(q_{1} q_{2}, q_{1} q_{2}, q_{3}, q_{1} q_{3}\right)=\theta_{4}\left(q_{1} q_{2}, q_{1} q_{2}, q_{1} q_{3}, q_{3}\right)$.)

The dual of the dual of $\theta_{r}\left(a_{1}, \ldots, a_{r}\right)$ is just $\theta_{r}\left(a_{1}, \ldots, a_{r}\right)$.

Under duality, a subset $S$ of $\left\{a_{1}, \ldots, a_{r}\right\}$ for which $\operatorname{gcd}(S)>1$ corresponds to a divisor $d>1$ of one of the $b_{j}$, and $(-1)^{|S|}=(-1)^{\omega(d)}=\mu(d)$. Hence

$$
\sum_{S \subseteq\left\{a_{1}, \ldots, a_{r}\right\},|S| \geq 2, \operatorname{gcd}(S)>1}(-1)^{|S|}=\sum_{d \mid b_{j} \text { for some } j, \omega(d) \geq 2} \mu(d) .
$$

Comparing (5) and (6) we have

$$
\operatorname{trace}\left(\theta_{n}\left(b_{1}, \ldots, b_{n}\right)\right)=n-r+\operatorname{trace}\left(\theta_{r}\left(a_{1}, \ldots, a_{r}\right)\right) .
$$

For an example of the usefulness of this, one can check from, e.g., (6) that for negative trace we must have $r \geq 6$. Duality immediately tells us that for negative trace (assuming (7) and (8)) we must have $n \geq 6$. This will be pursued further in a second paper, where it will be shown that we need $n \geq 8$, which is a best-possible bound.

4. More minimality conditions. Deleting all appearances of any $p_{i}$ $(1 \leq i \leq n)$ from $a_{1}, \ldots, a_{r}$ will reduce the degree, by (2). In seeking minimal 
degree with negative trace, we may therefore impose a third minimality condition, in addition to (7) and (8):

$$
\begin{aligned}
& \text { Deleting all appearances of any } p_{i} \text { will } \\
& \text { produce a Pisot number with larger trace. }
\end{aligned}
$$

Of course, we cannot delete all appearances of $p_{i}$ if this would reduce $r$ to 0 or 1 , or to 2 with $a_{1}=a_{2}=2$. Any such primes $p_{i}$ will be excluded from consideration in checking (10): we only delete deletable primes.

Dually we insist that

Deleting any $a_{i}$ will produce a

Pisot number with larger trace.

Again, if $r=2$ then we deem that (11) is satisfied although we cannot delete any $a_{i}$; or if $r=3$ then we only consider deletions which do not result in $r=a_{1}=a_{2}=2$ (if any).

Note that deleting an $a_{i}$ may not decrease the degree. Certainly the degree is never increased, but it will be unchanged if $a_{i}$ divides some other $a_{j}$. In this case the trace would be decreased by 1 , using (3). Thus (11) implies that

$$
\begin{aligned}
& \text { No } a_{i} \text { divides any other } a_{j} \text {. } \\
& \text { (Unless } r=2 \text {, or } r=3 \text { and } a_{1}=a_{2}=a_{3}=2 \text {.) }
\end{aligned}
$$

We label this as a new condition, for convenience, although as remarked it follows from (11). In effect we are insisting that amongst elements of $U$ with negative trace and minimal degree, we seek those with smallest (most negative) trace.

As a final minimality condition, we consider the effect of permuting the primes $p_{1}, \ldots, p_{n}$ which divide any of $a_{1}, \ldots, a_{r}$. This leaves the trace unchanged, but may change the degree, so we insist that:

No permutation of $p_{1}, \ldots, p_{n}$ will lower the degree.

Definition. If $\theta_{r}\left(a_{1}, \ldots, a_{r}\right)$ satisfies $(7),(8),(10)$ and (11) (and hence also (12)), and has negative trace, then we say that $\theta_{r}\left(a_{1}, \ldots, a_{r}\right)$ has a locally minimal pattern of primes. If also (13) holds, then we say that $\theta_{r}\left(a_{1}, \ldots, a_{r}\right)$ is locally minimal.

In seeking minimal degree amongst elements of $U$ with negative trace, we may restrict to locally minimal elements.

5. The two main theorems. The following result is extremely useful, and immediately provides a nontrivial lower bound on the degrees of elements of $U$ with negative trace, although we shall not pursue this here. 
THEOREM 1. If $\theta_{r}\left(a_{1}, \ldots, a_{r}\right)$ has a locally minimal pattern of primes, then each $a_{i}$ is divisible by at least four primes.

We shall see that four is best-possible: indeed there are locally minimal elements of $U$ for which each $a_{i}$ is divisible by exactly four primes.

From the proof, we isolate the following lemma, which will prove useful in constructing families of Pisot numbers with negative trace.

Lemma. Let $\theta_{r}\left(a_{1}, \ldots, a_{r}\right) \in U$. Let $p_{1}, \ldots, p_{s}$ be the distinct primes dividing $a_{1}$. For each $T \subseteq\{1, \ldots, s\}$, let $S_{T}$ be the subset of $\left\{a_{2}, \ldots, a_{r}\right\}$ containing those $a_{i}(2 \leq i \leq r)$ which are divisible by all the primes in $T$ :

$$
S_{T}=\left\{a_{i}: 2 \leq i \leq r, p_{j} \mid a_{i} \text { for all } j \in T\right\} .
$$

Then

$$
\begin{aligned}
& \operatorname{trace}\left(\theta_{r}\left(a_{1}, \ldots, a_{r}\right)\right) \\
& =\sum_{\emptyset \neq T \subseteq\{1, \ldots, s\}, S_{T} \neq \emptyset}(-1)^{|T|+1}+\sum_{S \subseteq\left\{a_{2}, \ldots, a_{r}\right\},|S| \geq 2, \operatorname{gcd}(S)>1}(-1)^{|S|} .
\end{aligned}
$$

Proof. We use (5), and split the sum as $\Sigma_{1}+\Sigma_{2}$, where

$$
\begin{aligned}
& \Sigma_{1}=\sum_{S \subseteq\left\{a_{1}, \ldots, a_{r}\right\}, a_{1} \in S,|S| \geq 2, \operatorname{gcd}(S)>1}(-1)^{|S|}, \\
& \Sigma_{2}=\sum_{S \subseteq\left\{a_{2}, \ldots, a_{r}\right\},|S| \geq 2, \operatorname{gcd}(S)>1}(-1)^{|S|} .
\end{aligned}
$$

We may suppose that $a_{1}=p_{1} \ldots p_{s}$, since repeated prime factors in $a_{1}$ change neither $\Sigma_{1}$ nor $\Sigma_{2}$.

For each subset $S \subseteq\left\{a_{1}, \ldots, a_{r}\right\}$ such that $a_{1} \in S,|S| \geq 2$ and $\operatorname{gcd}(S)>$ 1 , we consider those nonempty $T$ contained in $\{1, \ldots, s\}$ such that $\prod_{i \in T} p_{i}$ divides $\operatorname{gcd}(S)$ (equivalently, $S-\left\{a_{1}\right\} \subseteq S_{T}$ ). For such $S$ we have

$$
\sum_{T \neq \emptyset, \prod_{i \in T} p_{i} \mid \operatorname{gcd}(S)}(-1)^{|T|+1}=1,
$$

hence

$$
\begin{aligned}
\Sigma_{1} & =\sum_{S}(-1)^{|S|} \sum_{T \neq \emptyset, \prod_{i \in T} p_{i} \mid \operatorname{gcd}(S)}(-1)^{|T|+1} \\
& =\sum_{T \neq \emptyset}(-1)^{|T|+1} \sum_{S-\left\{a_{1}\right\} \subseteq S_{T},|S| \geq 2, a_{1} \in S}(-1)^{|S|} \\
& =\sum_{T \neq \emptyset, S_{T} \neq \emptyset}(-1)^{|T|+1},
\end{aligned}
$$

as desired. 
Note that $\Sigma_{2}$ is the trace of the Pisot number obtained by deleting $a_{1}$. Of course there is nothing special about $a_{1}$, and the Lemma tells us how to compute the change of trace if we add or delete any $a_{i}$.

For example, with $\theta_{3}(6,10,15)$, we have $a_{1}=6=2 \times 3, S_{\{2\}}=\{10\}$, $S_{\{3\}}=\{15\}, S_{\{2,3\}}=\emptyset$, and

$$
\sum_{T \neq \emptyset, S_{T} \neq \emptyset}(-1)^{|T|+1}=(-1)^{|\{2\}|+1}+(-1)^{|\{3\}|+1}=1+1=2,
$$

so we have

$$
\operatorname{trace}\left(\theta_{3}(6,10,15)\right)=2+\operatorname{trace}\left(\theta_{2}(10,15)\right)=3 .
$$

Proof of Theorem 1. Suppose that $\theta_{r}\left(a_{1}, \ldots, a_{r}\right)$ has a locally minimal pattern of primes. By (7) we have $a_{1}=p_{1} \ldots p_{s}$ for distinct primes $p_{1}, \ldots, p_{s}$. For negative trace, one readily checks from (3) or (5) that $r>3$, so that $\theta_{r-1}\left(a_{2}, \ldots, a_{r}\right) \in U$ and has larger trace. By the Lemma,

$$
\Sigma_{1}=\sum_{\emptyset \neq T \subseteq\{1, \ldots, s\}, S_{T} \neq \emptyset}(-1)^{|T|+1}<0,
$$

where $S_{T}$ is defined by (14).

Note that by (10), $S_{\left\{p_{i}\right\}} \neq \emptyset$ for any $i$, else we could delete $p_{i}$ without changing the trace.

If $s=1$, then

$$
\Sigma_{1}=\sum_{T=\left\{p_{1}\right\}}(-1)^{|T|+1}=1 .
$$

If $s=2$, then either

$$
\Sigma_{1}=\sum_{T \in\left\{\left\{p_{1}\right\},\left\{p_{2}\right\}\right\}}(-1)^{|T|+1}=2,
$$

or

$$
\Sigma_{1}=\sum_{T \in\left\{\left\{p_{1}\right\},\left\{p_{2}\right\},\left\{p_{1}, p_{2}\right\}\right\}}(-1)^{|T|+1}=1 .
$$

If $s=3$, then if $S_{\left\{p_{1}, p_{2}, p_{3}\right\}} \neq \emptyset$ we have $\Sigma_{1}=3-3+1=1$, else $\Sigma_{1} \geq 3-3=0$.

Hence we must have $s \geq 4$. There is nothing special about $a_{1}$, so each $a_{i}$ must be divisible by at least four primes.

One can also prove Theorem 1 using (3), rather than (5). I have chosen to go via (5) because the Lemma will be useful later.

If $a_{1}=p_{1} p_{2} p_{3} p_{4}$, then we can be rather precise about the possible ways in which the primes $p_{1}, p_{2}, p_{3}, p_{4}$ appear amongst the other $a_{i}$ :

ThEOREM 2. Suppose that $\theta_{r}\left(a_{1}, \ldots, a_{r}\right)$ has a locally minimal pattern of primes, and that $a_{1}=p_{1} p_{2} p_{3} p_{4}$ with $p_{1}, p_{2}, p_{3}, p_{4}$ primes. Then, after relabelling the primes and the $a_{i}$ if necessary, we have one of three cases: 
Case A:

$$
\begin{aligned}
& a_{1}=p_{1} \cdot p_{2} \cdot p_{3} \cdot p_{4}, \\
& a_{2}=p_{1} \cdot p_{2} \cdot b_{2}, \\
& a_{3}=p_{1} \cdot p_{3} \cdot b_{3}, \\
& a_{4}=p_{1} \cdot p_{4} \cdot b_{4}, \\
& a_{5}=p_{2} \cdot p_{3} \cdot b_{5}, \\
& a_{6}=p_{2} \cdot p_{4} \cdot b_{6}, \\
& a_{7}=p_{3} \cdot p_{4} \cdot b_{7},
\end{aligned}
$$

with $b_{2}, \ldots, b_{7}$ coprime to $a_{1}$. We may have $r>7$, but no further $a_{i}$ can be divisible by any three of $p_{1}, p_{2}, p_{3}, p_{4}$.

Case B:

$$
\begin{aligned}
& a_{1}=p_{1} \cdot p_{2} \cdot p_{3} \cdot p_{4}, \\
& a_{2}=p_{1} \cdot p_{2} \cdot b_{2}, \\
& a_{3}=p_{1} \cdot p_{3} \cdot b_{3}, \\
& a_{4}=p_{1} \cdot p_{4} \cdot b_{4}, \\
& a_{5}=p_{2} \cdot p_{3} \cdot b_{5}, \\
& a_{6}=p_{2} \cdot p_{4} \cdot b_{6},
\end{aligned}
$$

with $b_{2}, \ldots, b_{6}$ coprime to $a_{1}$. Each further $a_{i}$ is divisible by at most two of $p_{1}, p_{2}, p_{3}, p_{4}$, and is not divisible by $p_{3} p_{4}$.

Case C:

$$
\begin{aligned}
& a_{1}=p_{1} \cdot p_{2} \cdot p_{3} \cdot p_{4}, \\
& a_{2}=p_{1} \cdot p_{2} \cdot p_{3} \cdot b_{2}, \\
& a_{3}=p_{1} \cdot p_{4} \cdot b_{3}, \\
& a_{4}=p_{2} \cdot p_{4} \cdot b_{4}, \\
& a_{5}=p_{3} \cdot p_{4} \cdot b_{5},
\end{aligned}
$$

with $b_{2}, \ldots, b_{5}$ coprime to $a_{1}$. Each further $a_{i}$ is divisible by at most two of $p_{1}, p_{2}, p_{3}, p_{4}$, except that we allow divisibility by $p_{1} p_{2} p_{3}$.

In Case $A$, deleting $a_{1}$ increases the trace by 2 ; in Cases $B$ and $C$, deleting $a_{1}$ increases the trace by 1 .

Proof. As in the proof of Theorem 1, we have $S_{\left\{p_{i}\right\}} \neq \emptyset$ for $i=1,2,3,4$.

Suppose first that $S_{\left\{p_{i}, p_{j}, p_{k}\right\}} \neq \emptyset$ for some triple of primes $\left(p_{i}, p_{j}, p_{k}\right)$, with $S_{T}$ defined by (14). Relabelling, we may assume that $S_{\left\{p_{1}, p_{2}, p_{3}\right\}} \neq \emptyset$. Then, with notation as in the Lemma,

$$
\Sigma_{1}=3-3+1+\sum_{p_{4} \in T, S_{T} \neq \emptyset}(-1)^{|T|+1},
$$


so we require

$$
\sum_{p_{4} \in T, S_{T} \neq \emptyset}(-1)^{|T|+1} \leq-2
$$

We can achieve this if and only if $S_{\left\{p_{1}, p_{4}\right\}}, S_{\left\{p_{2}, p_{4}\right\}}, S_{\left\{p_{3}, p_{4}\right\}}$ are all nonempty, and $S_{\left\{p_{i}, p_{j}, p_{4}\right\}}=\emptyset$ for any $1 \leq i<j<4$. This gives us Case C.

Next we have the possibility that no three of $p_{1}, p_{2}, p_{3}, p_{4}$ divide any one of $a_{2}, \ldots, a_{r}$. To achieve $\Sigma_{1}<0$ we need $S_{\left\{p_{i}, p_{j}\right\}} \neq \emptyset$ for either 5 or 6 of the possible pairs $1 \leq i<j \leq 4$, giving Case B or Case A respectively.

COROLlary. If $\theta_{r}\left(a_{1}, \ldots, a_{r}\right)$ has a locally minimal pattern of primes and if each $p_{i}$ divides exactly three of the $a_{i}$, then each $a_{i}$ is divisible by at least five primes.

We shall see that "five" is best-possible.

Pr o of. Examining the patterns in Cases A, B, C of Theorem 2, we see that at least one of $p_{1}, p_{2}, p_{3}, p_{4}$ divides at least four of the $a_{i}$.

6. The symmetry group, and some locally minimal examples. Given a locally minimal pattern of $n$ primes, we still have $n$ ! permutations of the first $n$ primes to consider in order to find a locally minimal Pisot number. Exploiting symmetry speeds this search.

Definition. The symmetry group of $\theta_{r}\left(a_{1}, \ldots, a_{r}\right)$ is the group consisting of those permutations of $p_{1}, \ldots, p_{n}$ which induce permutations of $a_{1}, \ldots, a_{r}$ (and so fix the Pisot number).

For example, let us consider the pattern given in [1] with trace -5 . This took $r=6, n=20$, with each prime dividing exactly three of the $a_{i}$ : all $\left(\begin{array}{l}6 \\ 3\end{array}\right)=20$ triples being covered. By $(5)$, trace $=\left(\begin{array}{l}6 \\ 2\end{array}\right)-\left(\begin{array}{l}6 \\ 3\end{array}\right)=-5$.

This pattern is locally minimal, but we can delete any four of the $p_{i}$ and still have a negative trace.

It seems at first natural to delete four primes as symmetrically as possible, giving the following locally minimal pattern with $r=6, n=16$ :

$$
\begin{aligned}
& a_{1}=p_{2} \cdot p_{3} \cdot p_{6} \cdot p_{7} \cdot p_{8} \cdot p_{11} \cdot p_{13} \cdot p_{14}, \\
& a_{2}=p_{2} \cdot p_{4} \cdot p_{5} \cdot p_{6} \cdot p_{8} \cdot p_{14} \cdot p_{15} \cdot p_{16}, \\
& a_{3}=p_{2} \cdot p_{4} \cdot p_{5} \cdot p_{9} \cdot p_{10} \cdot p_{11} \cdot p_{12} \cdot p_{13}, \\
& a_{4}=p_{1} \cdot p_{4} \cdot p_{7} \cdot p_{8} \cdot p_{10} \cdot p_{12} \cdot p_{13} \cdot p_{15}, \\
& a_{5}=p_{1} \cdot p_{3} \cdot p_{6} \cdot p_{9} \cdot p_{10} \cdot p_{11} \cdot p_{15} \cdot p_{16}, \\
& a_{6}=p_{1} \cdot p_{3} \cdot p_{5} \cdot p_{7} \cdot p_{9} \cdot p_{12} \cdot p_{14} \cdot p_{16}
\end{aligned}
$$

The symmetry group has order 24 , and is isomorphic to $S_{4}$. It is generated by

$$
\left(p_{1} p_{16}\right)\left(p_{2} p_{13}\right)\left(p_{3} p_{9}\right)\left(p_{4} p_{8}\right)\left(p_{5} p_{7}\right)\left(p_{6} p_{10}\right)\left(p_{12} p_{14}\right)
$$




$$
\left(p_{1} p_{8}\right)\left(p_{2} p_{9}\right)\left(p_{3} p_{14}\right)\left(p_{4} p_{10}\right)\left(p_{5} p_{11}\right)\left(p_{6} p_{16}\right)\left(p_{12} p_{13}\right),
$$

and

$$
\left(p_{1} p_{12}\right)\left(p_{2} p_{6}\right)\left(p_{3} p_{13}\right)\left(p_{4} p_{16}\right)\left(p_{5} p_{15}\right)\left(p_{8} p_{14}\right)\left(p_{9} p_{10}\right) \text {. }
$$

For this case, I shall give a proof that this is indeed the symmetry group: in later (simpler!) cases I shall leave the justification as an exercise.

Certainly the above three permutations of $p_{1}, \ldots, p_{16}$ induce permutations of $a_{1}, \ldots, a_{6}$, namely

$$
\begin{aligned}
& \left(\begin{array}{ll}
a_{1} & a_{3}
\end{array}\right)\left(\begin{array}{ll}
a_{2} & a_{4}
\end{array}\right), \\
& \left(\begin{array}{ll}
a_{1} & a_{6}
\end{array}\right)\left(\begin{array}{ll}
a_{2} & a_{5}
\end{array}\right),
\end{aligned}
$$

and

$$
\left(a_{4} a_{6}\right)\left(a_{3} a_{5}\right) \text {. }
$$

Also they induce permutations of $\left\{p_{5}, p_{7}, p_{11}, p_{15}\right\}$, namely $\left(p_{5} p_{7}\right),\left(p_{5} p_{11}\right)$, and $\left(p_{5} p_{15}\right)$. These three transpositions generate the full symmetric group on $\left\{p_{5}, p_{7}, p_{11}, p_{15}\right\}$, so our symmetry group, $G$ say, contains a subgroup isomorphic to $S_{4}$.

It is enough now to show that any element of $G$ which fixes each of $p_{5}, p_{7}, p_{11}, p_{15}$, fixes every prime. First note that each $a_{i}$ is divisible by a uniquely-determined pair of primes from $\left\{p_{5}, p_{7}, p_{11}, p_{15}\right\}$, so any element of $G$ fixing each of $p_{5}, p_{7}, p_{11}, p_{15}$ fixes each of $a_{1}, \ldots, a_{6}$. But each $p_{i}$ divides a uniquely-determined triple of $a_{1}, \ldots, a_{6}$, so any element of $G$ fixing $a_{1}, \ldots, a_{6}$ must fix $p_{1}, \ldots, p_{16}$, and we are done.

A pleasing geometrical interpretation of this symmetry group was supplied by Chris Smyth and Elmer Rees. We can view $a_{1}, \ldots, a_{6}$ as the edges of a tetrahedron. Each $p_{i}$ appears in three edges, and the four missing triples can be taken to correspond to the four faces of the tetrahedron (or their complements). The symmetry group then corresponds to permutations of the vertices of the tetrahedron.

In principle, utilising this symmetry group reduces the search for a locally minimal Pisot number with this pattern of primes by a factor of 24 . In practice, I found it easier to fix $p_{1}$ and $p_{2}$, then loop through all 14 ! possibilities for $p_{3}, \ldots, p_{16}$. Under the action of $G$ there are twelve orbits for the ordered pair $\left(p_{1}, p_{2}\right)$, so I searched through $12 \times 14$ ! possibilities, gaining a factor of 20 rather than 24 . The search took two weeks (rather than forty) on my home PC. The minimal degree is 34250586162 , achieved, for example, when

$$
\left(p_{1}, \ldots, p_{16}\right)=(7,17,47,29,2,13,3,23,31,19,5,53,37,43,11,41) .
$$

In fact one can do a little better (also noted by Chris Smyth) by deleting four triples from the twenty asymmetrically, deleting all those dividing both $a_{1}$ and $a_{2}$. This leaves $\operatorname{gcd}\left(a_{1}, a_{2}\right)=1$, allowing deletion of a further prime, 
giving trace $14-15=-1$ (using (5)). There are two essentially distinct ways of deleting this fifth prime, the first of which shows that the "five" in the Corollary to Theorem 2 is best-possible.

The first possibility is:

$$
\begin{aligned}
& a_{1}=p_{1} \cdot p_{2} \cdot p_{3} \cdot p_{4} \cdot p_{5}, \\
& a_{2}=p_{6} \cdot p_{7} \cdot p_{8} \cdot p_{9} \cdot p_{10} \cdot p_{11}, \\
& a_{3}=p_{1} \cdot p_{3} \cdot p_{6} \cdot p_{7} \cdot p_{11} \cdot p_{12} \cdot p_{13} \cdot p_{15}, \\
& a_{4}=p_{4} \cdot p_{5} \cdot p_{6} \cdot p_{8} \cdot p_{10} \cdot p_{12} \cdot p_{14} \cdot p_{15}, \\
& a_{5}=p_{2} \cdot p_{3} \cdot p_{4} \cdot p_{7} \cdot p_{8} \cdot p_{9} \cdot p_{13} \cdot p_{14} \cdot p_{15}, \\
& a_{6}=p_{1} \cdot p_{2} \cdot p_{5} \cdot p_{9} \cdot p_{10} \cdot p_{11} \cdot p_{12} \cdot p_{13} \cdot p_{14} .
\end{aligned}
$$

The symmetry group has order 4 , isomorphic to $C_{2} \times C_{2}$. The minimal degree is 12160477837 .

Moving $p_{15}$ from $a_{5}$ to $a_{1}$ gives the second possibility:

$$
\begin{aligned}
& a_{1}=p_{1} \cdot p_{2} \cdot p_{3} \cdot p_{4} \cdot p_{5} \cdot p_{15}, \\
& a_{2}=p_{6} \cdot p_{7} \cdot p_{8} \cdot p_{9} \cdot p_{10} \cdot p_{11}, \\
& a_{3}=p_{1} \cdot p_{3} \cdot p_{6} \cdot p_{7} \cdot p_{11} \cdot p_{12} \cdot p_{13} \cdot p_{15}, \\
& a_{4}=p_{4} \cdot p_{5} \cdot p_{6} \cdot p_{8} \cdot p_{10} \cdot p_{12} \cdot p_{14} \cdot p_{15}, \\
& a_{5}=p_{2} \cdot p_{3} \cdot p_{4} \cdot p_{7} \cdot p_{8} \cdot p_{9} \cdot p_{13} \cdot p_{14}, \\
& a_{6}=p_{1} \cdot p_{2} \cdot p_{5} \cdot p_{9} \cdot p_{10} \cdot p_{11} \cdot p_{12} \cdot p_{13} \cdot p_{14}
\end{aligned}
$$

There are more symmetries here: 12 of them, with the symmetry group isomorphic to $S_{3} \times C_{2}$. The minimal degree is 7627134993 .

Even with $r=6$ one can do much better, by allowing the $p_{j}$ to divide more than three of the $a_{i}$. Consider the following ten-prime pattern:

$$
\begin{aligned}
& a_{1}=p_{1} \cdot p_{2} \cdot p_{4} \cdot p_{9} \cdot p_{10}, \\
& a_{2}=p_{1} \cdot p_{2} \cdot p_{5} \cdot p_{6} \cdot p_{7} \cdot p_{8}, \\
& a_{3}=p_{1} \cdot p_{3} \cdot p_{5} \cdot p_{6} \cdot p_{7}, \\
& a_{4}=p_{1} \cdot p_{3} \cdot p_{4} \cdot p_{8} \cdot p_{9} \cdot p_{10}, \\
& a_{5}=p_{2} \cdot p_{3} \cdot p_{5} \cdot p_{7} \cdot p_{9}, \\
& a_{6}=p_{2} \cdot p_{3} \cdot p_{4} \cdot p_{6} \cdot p_{8} \cdot p_{10} .
\end{aligned}
$$

All 15 pairs $\left(a_{i}, a_{j}\right)$ have gcd $>1$, as do 19 of the 20 triples (the missing one being $\left.\left(a_{1}, a_{3}, a_{5}\right)\right)$. There are three primes appearing four times, giving trace $15-19+3=-1$ (using (5)). The symmetry group is $S_{3}$, and the minimal degree is 1106669 , achieved, for example, when $\left(p_{1}, \ldots, p_{10}\right)=$ $(3,5,7,29,19,13,17,2,23,11)$. 
7. Constructing smaller examples. We can use the patterns of Theorem 2 , and the result of the Lemma, to try to build locally minimal patterns with smaller degree.

To accommodate Case $\mathrm{A}$ of Theorem 2, we need $r \geq 7$, and the total number of primes, counting with multiplicity, must be at least 28 . The following remarkable pattern would be locally minimal, if only it had negative trace!

$$
\begin{aligned}
& a_{1}=p_{1} \cdot p_{2} \cdot p_{3} \cdot p_{4}, \\
& a_{2}=p_{1} \cdot p_{2} \cdot p_{5} \cdot p_{6}, \\
& a_{3}=p_{1} \cdot p_{3} \cdot p_{5} \cdot p_{7}, \\
& a_{4}=p_{1} \cdot p_{4} \cdot p_{6} \cdot p_{7}, \\
& a_{5}=p_{2} \cdot p_{3} \cdot p_{6} \cdot p_{7}, \\
& a_{6}=p_{2} \cdot p_{4} \cdot p_{5} \cdot p_{7}, \\
& a_{7}=p_{3} \cdot p_{4} \cdot p_{5} \cdot p_{6} .
\end{aligned}
$$

This satisfies all the minimality conditions (7), (8), (10), (11), but has trace $21-28+7=0$ (using (5)). The pattern is self-dual. Each pair of the $a_{i}$ is divisible by exactly two of the $p_{j}$. With only 28 of the 35 possible triples covered, we can modify this pattern to give trace -1 by adding an eighth prime, dividing, say, $a_{1}, a_{2}$ and $a_{7}$. Relabelling we get a locally minimal pattern with a total of only 31 primes (the record: presumably optimal):

$$
\begin{aligned}
& a_{1}=p_{1} \cdot p_{2} \cdot p_{3} \cdot p_{4} \cdot p_{8}, \\
& a_{2}=p_{1} \cdot p_{2} \cdot p_{5} \cdot p_{6} \cdot p_{8}, \\
& a_{3}=p_{3} \cdot p_{4} \cdot p_{5} \cdot p_{6} \cdot p_{8}, \\
& a_{4}=p_{1} \cdot p_{3} \cdot p_{5} \cdot p_{7}, \\
& a_{5}=p_{1} \cdot p_{4} \cdot p_{6} \cdot p_{7}, \\
& a_{6}=p_{2} \cdot p_{3} \cdot p_{6} \cdot p_{7}, \\
& a_{7}=p_{2} \cdot p_{4} \cdot p_{5} \cdot p_{7} .
\end{aligned}
$$

The trace is $21-29+7=-1$ (using (5)). The symmetry group is isomorphic to $S_{4}$ (permuting $a_{4}, a_{5}, a_{6}, a_{7}$, and exhibiting $S_{3}$ as a quotient group, permuting $a_{1}, a_{2}, a_{3}$ ). The minimal degree for this pattern is 69213 , achieved when $a_{1}, \ldots, a_{7}$ is some permutation of $14586,15470,19635,570$, $2926,5187,13585$.

It would be nice to have each $a_{i}$ divisible by only 4 primes. With $r=7$, 8 , or 9 , this is impossible. But for $r=10$ we can achieve it. The simplest construction takes two copies of Case $\mathrm{A}$ in Theorem 2, based on primes $\left\{p_{1}, p_{2}, p_{3}, p_{4}\right\}$ and $\left\{p_{5}, p_{6}, p_{7}, p_{8}\right\}$, and glues them together to give a self- 
dual pattern with $r=n=8$ :

$$
\begin{aligned}
& a_{1}=p_{1} \cdot p_{2} \cdot p_{3} \cdot p_{4}, \\
& a_{2}=p_{1} \cdot p_{2} \cdot p_{5} \cdot p_{6}, \\
& a_{3}=p_{1} \cdot p_{3} \cdot p_{5} \cdot p_{7}, \\
& a_{4}=p_{1} \cdot p_{4} \cdot p_{6} \cdot p_{7}, \\
& a_{5}=p_{2} \cdot p_{3} \cdot p_{5} \cdot p_{8}, \\
& a_{6}=p_{2} \cdot p_{4} \cdot p_{6} \cdot p_{8}, \\
& a_{7}=p_{3} \cdot p_{4} \cdot p_{7} \cdot p_{8}, \\
& a_{8}=p_{5} \cdot p_{6} \cdot p_{7} \cdot p_{8} .
\end{aligned}
$$

Here the trace is $24-32+8=0$ (using (5)). Using the Lemma, we see that although there are no values of $a_{9}$ with $\omega\left(a_{9}\right)=4$ which would make the trace negative, there are several that preserve trace 0 , such as

$$
a_{9}=p_{1} \cdot p_{2} \cdot p_{7} \cdot p_{8},
$$

giving $\Sigma_{1}=4-4=0\left(S_{\left\{p_{1}, p_{8}\right\}}=S_{\left\{p_{2}, p_{7}\right\}}=\emptyset\right)$, in the notation of the Lemma. And now if we add, for example,

$$
a_{10}=p_{1} \cdot p_{3} \cdot p_{6} \cdot p_{8}
$$

we achieve $\Sigma_{1}=4-5=-1$, and $\operatorname{trace}\left(\theta_{10}\left(a_{1}, \ldots, a_{10}\right)\right)=-1$. In terms of (5) we have trace $=41-80+52-16+2=-1$. Although we have a total of forty primes, we can achieve a smaller degree than before, helped by each $a_{i}$ being divisible by only 4 primes. The symmetry group is nonabelian of order 16 , and the minimal degree is 25125 , achieved by

$$
\begin{aligned}
a_{1} & =2 \cdot 5 \cdot 7 \cdot 17, \\
a_{2} & =2 \cdot 5 \cdot 11 \cdot 19, \\
a_{3} & =2 \cdot 7 \cdot 13 \cdot 19, \\
a_{4} & =2 \cdot 11 \cdot 13 \cdot 17, \\
a_{5} & =3 \cdot 5 \cdot 7 \cdot 19, \\
a_{6} & =3 \cdot 5 \cdot 11 \cdot 17, \\
a_{7} & =3 \cdot 7 \cdot 13 \cdot 17, \\
a_{8} & =3 \cdot 11 \cdot 13 \cdot 19, \\
a_{9} & =2 \cdot 3 \cdot 7 \cdot 11, \\
a_{10} & =2 \cdot 3 \cdot 5 \cdot 13 .
\end{aligned}
$$

We can do marginally better. Consider the first locally minimal pattern of this section, with $r=7, n=8$, and 31 primes. The dual pattern has 
$r=8, n=7$, and trace $8-7+(-1)=0$, using $(9)$ :

$$
\begin{aligned}
& a_{1}=p_{1} \cdot p_{2} \cdot p_{3} \cdot p_{4}, \\
& a_{2}=p_{1} \cdot p_{2} \cdot p_{5} \cdot p_{6}, \\
& a_{3}=p_{1} \cdot p_{3} \cdot p_{5} \cdot p_{7}, \\
& a_{4}=p_{1} \cdot p_{4} \cdot p_{6} \cdot p_{7}, \\
& a_{5}=p_{2} \cdot p_{3} \cdot p_{6} \cdot p_{7}, \\
& a_{6}=p_{2} \cdot p_{4} \cdot p_{5} \cdot p_{7}, \\
& a_{7}=p_{3} \cdot p_{4} \cdot p_{5} \cdot p_{6}, \\
& a_{8}=p_{1} \cdot p_{2} \cdot p_{7}
\end{aligned}
$$

(after some relabelling). We can replace $a_{8}$ by

$$
a_{8}=p_{1} \cdot p_{2} \cdot p_{7} \cdot p_{8}
$$

without changing the trace. Now adding

$$
a_{9}=p_{1} \cdot p_{4} \cdot p_{5} \cdot p_{8}
$$

preserves trace $0: \Sigma_{1}=4-4=0$. Finally, adding

$$
a_{10}=p_{3} \cdot p_{4} \cdot p_{7} \cdot p_{8}
$$

gives us trace $-1: \Sigma_{1}=4-5=-1$. In terms of (5), we have trace $=$ $42-86+61-21+3=-1$.

The gain over the previous pattern is that now we have three primes appearing 6 times, rather than two, and one prime appearing only 3 times. This allows us to have more smaller primes. The symmetry group is $S_{3}$, and the minimal degree is 23837 , achieved when $a_{1}, \ldots, a_{10}$ is some permutation of 390, 462, 1190, 1938, 1995, 2090, 2805, 4641, 4862, 5005.

\section{Reference}

[1] J. F. McKee, P. Rowlinson and C. J. Smyth, Salem numbers and Pisot numbers from stars, in: Number Theory in Progress, Vol. I, K. Györy, H. Iwaniec and J. Urbanowicz (eds.), de Gruyter, Berlin, 1999, 309-319.

Pembroke College

Oxford OX1 1DW

England

E-mail: mckee@maths.ox.ac.uk 